\title{
MIB-1 labeling index, Ki-67, is an indicator of invasive intraductal papillary mucinous neoplasm
}

\author{
TATSUO SHIMURA $^{1}$, YASUHIDE KOFUNATO ${ }^{2}$, RYO OKADA $^{2}$, REI YASHIMA $^{2}$, KOJI OKADA $^{3}$, \\ KENICHIRO ARAKI ${ }^{3}$, YASUO HOSOUCHI ${ }^{4}$, HIROYUKI KUWANO ${ }^{3}$ and SEIICHI TAKENOSHITA ${ }^{2}$
}

\author{
Departments of ${ }^{1}$ Cancer Biology and Electronics and ${ }^{2}$ Organ Regulatory Surgery, Fukushima Medical University, \\ Fukushima 960-1295; ${ }^{3}$ Department of General Surgical Science, Gunma University Graduate School of Medicine, \\ Gunma 371-8511; ${ }^{4}$ Department of Surgery and Laparoscopic Surgery, Gunma Prefecture \\ Saiseikai-Maebashi Hospital, Maebashi, Gunma 371-0821, Japan
}

Received March 11, 2016; Accepted May 9, 2016

DOI: $10.3892 / \mathrm{mco} .2016 .908$

\begin{abstract}
Despite strict criteria for the observation of intraductal papillary mucinous neoplasm (IPMN), it remains difficult to distinguish invasive IPMN from non-invasive IPMN. The aim of the present study was to identify an indicator of invasive IPMN. The present study retrospectively evaluated 53 patients ( 28 with non-invasive and 25 with invasive IPMN) who underwent resection of IPMN, and examined the usefulness of the MIB-1 labeling index as an indicator of invasive IPMN. The MIB-1 labeling indexes in patients with invasive IPMN were significantly higher compared with those with non-invasive IPMN $(\mathrm{P}<0.001)$. A receiver operating characteristic curve revealed that the area under the curve was 0.822 . These results suggested that a cut-off level for the MIB-1 labeling index should be set to $15.5 \%$ to distinguish invasive from non-invasive IPMN. A multivariate analysis using a logistic regression model revealed the MIB-1 labeling index (hazard ratio, 18.692; 95\% confidential interval, 4.171-83.760; $\mathrm{P}<0.001$ ) and the existence of mural nodules (hazard ratio, 6.187, 95\% confidential interval, 1.039-36.861; $\mathrm{P}=0.045$ ) were predictive factors for invasive IPMN. However, no statistically significant differences were observed between patients with a lower MIB-1 labeling index and patients with a higher MIB-1 labeling index ( $\mathrm{P}=0.798)$. The MIB-1 labeling index must be considered as a candidate for the classification of IPMN.
\end{abstract}

Correspondence to: Professor Tatsuo Shimura, Department of Cancer Biology and Electronics, Fukushima Medical University, 1 Hikarigaoka, Fukushima 960-1295, Japan

E-mail: tshimura@fmu.ac.jp

Abbreviations: IPMN, intraductal papillary mucinous neoplasm; PBS, phosphate-buffered saline

Key words: IPMN, MIB-1, Ki-67, invasive IPMN, mural nodule

\section{Introduction}

Since Ohashi et al (1) first described intraductal papillary mucinous neoplasm (IPMN) in 1982, IPMNs have become recognized as the most common of all cystic tumors of the pancreas, accounting for up to $70 \%$ (2). On the basis of the location of ductal involvement, IPMNs are divided into three groups: Main duct IPMN, branch duct IPMN and mixed type IPMN (3). The first International Consensus Guidelines for IPMN management were published in 2006 (3) and were later updated in 2012 (4). According to the guidelines, surgical resection is recommended for all main duct IPMNs due to the high risk of malignancy $(61.6 \%)$ and invasive carcinoma $(43.1 \%)(4,5)$. By contrast, the frequency of malignant and invasive IPMNs in branch duct IPMN were reported to be 25.5 and $17.7 \%$, respectively (4). The latest International Consensus Guidelines, however, described worrisome features of malignancy, including a cyst $>3 \mathrm{~cm}$, thickened and enhanced cyst walls, main pancreatic duct size 5-9 mm, non-enhancing mural nodule, abrupt change in caliber of duct with distal pancreatic atrophy and lymphadenopathy (4). No criterion has been proven accurate in predicting an invasive progression in main duct IPMN (6). Several previous studies described predictors of malignancy of main duct IPMN: Older age, more frequent incidence of jaundice and/or worsening of diabetes, $>15 \mathrm{~mm}$ dilatation of the main pancreatic duct and a mural nodule $(5,7)$. However, $29 \%$ of the patients with malignant main duct IPMN were asymptomatic (5), and those with smaller main duct dilatation and no mural nodule had invasive carcinomas (7). Previously, a number of additional predictors of malignancy in branch duct IPMNs were reported: Elevated tumor markers, an increase of cyst size over time, family history, multifocal IPMN or obesity (8-12).

An unsettled definition of IPMN malignancy makes comparison of the described data difficult. Certain reports included cases with carcinoma in situ into those of malignant IPMNs, while other studies enrolled patients with invasive IPMN only into those of malignant disease. The new International Consensus Guidelines described carcinoma in situ as high-grade dysplasia (4).

By contrast, the MIB-1 index has been used for diagnosing malignancy in other diseases. In neuroendocrine tumors, those 
with an MIB-1 labeling index of $<2 \%$ are classified as G1, and those with an index between 2 and $20 \%$ as G2. Tumors with an index of $>20 \%$ are classified as neuroendocrine carcinoma (13). In early breast carcinoma, patients with a high MIB-1 labeling index have a poor prognosis (14). As for IPMNs, several reports have presented data of the MIB-1 labeling index (15-22). However, confusing criteria for the definition of malignant IPMNs prevent us from comparing these results.

The aim of the present study was to identify clinical and pathologic features of invasive IPMN using our cohort approach that simply classifies patients into two groups: Non-invasive and invasive IPMN. The present study also aimed to identify the role of the MIB-1 labeling index as an indicator of invasive IPMNs.

\section{Materials and methods}

Patients. A total of 53 patients with IPMNs who underwent resection of tumors between 2000 and 2010 were enrolled, in accordance with the guidelines for informed consent and approval from the Ethics Committee of our institute. Of these patients, 28 patients exhibited non-invasive IPMN, including three patients with carcinoma in situ of IPMN, and 25 patients with invasive IPMN. The neoplasms were classified into non-invasive IPMNs and invasive IPMNs. Minimally invasive IPMNs were classified into invasive IPMNs. The neoplasms in the head, neck or uncinate process of the pancreas were treated with pancreaticoduodenectomy, and neoplasms in the pancreatic body or tail were treated with open or laparoscopic distal pancreatectomy accordingly.

Analysis on factors for invasive IPMN. As for the clinical features in determining predictive factors for invasive IPMN, age, gender, tumor size, type of involved duct (main or mixed type vs. branch duct), with or without symptoms, dilatation of the main duct and a mural nodule in pre-operative imaging modalities, and the MIB-1 labeling index were investigated.

Immunohistochemical analysis. The MIB-1 labeling index was assessed by immunohistochemistry using an avidin-biotin-peroxidase complex method. Formalin-fixed, paraffin-embedded tissue samples were cut into $4 \mu \mathrm{m}$-thick sections. The sections were deparaffinized in xylene and rehydrated through a series of decreasing alcohol concentrations. Following this, they were rinsed three times in phosphate-buffered saline (PBS), and the sections were immersed in an absolute methanol solution containing $0.3 \% \mathrm{H}_{2} \mathrm{O}_{2}$ for $30 \mathrm{~min}$ at room temperature to inhibit endogenous peroxidase. Antigens were retrieved by autoclaving sections on slides in $0.01 \mathrm{M}$ (pH 6.0) citrate buffer for $10 \mathrm{~min}$. After rinsing in PBS, the sections were incubated with monoclonal mouse anti-human antibody against Ki-67 (Clone, MIB-1; cat. no. M724001; Dako, Tokyo, Japan; $1: 50$ ) overnight at $4^{\circ} \mathrm{C}$. A further wash in PBS was followed by treatment with peroxidase-labeled anti-mouse antibody (Histofine Simple Stain Max-PO (M); Nichirei, Tokyo, Japan) as the secondary antibody for $30 \mathrm{~min}$ at room temperature. The staining was visualized with diaminobenzidine. Immunohistochemical evaluations were performed with a microscope (magnification, $\mathrm{x} 100$ ). A total of 1,000 tumor cells were counted to assess positive staining,

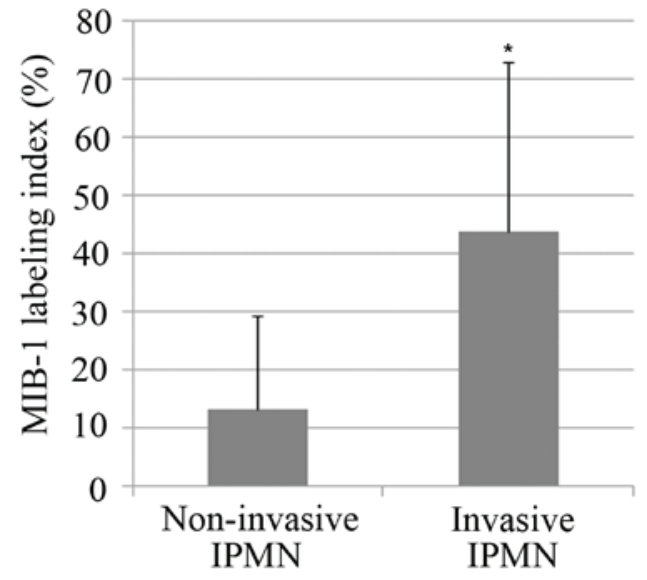

Figure 1. MIB-1 labeling index in patients with non-invasive and invasive IPMN. The MIB-1 labeling index was $13.4 \pm 15.8$ and $42.4 \pm 30.3$ in patients with non-invasive and invasive IPMN, respectively. The data are expressed as the mean \pm standard deviation $\left({ }^{*} \mathrm{P}<0.001\right)$. IPMN, intraductal papillary mucinous neoplasm.

and the percentages of positively stained cells were determined as the MIB-1 labeling index.

Statistical analysis. Categorical variables were evaluated by either the $\chi^{2}$ or Fisher's exact test. Predictors of invasive IPMNs were determined with univariate and multivariate analyses using a logistic regression model. To assess the performance characteristics of the MIB-1 labeling index, receiver operating characteristic curves were generated and the area under the curve was calculated. The survival time was observed between the date of surgery and date of the last follow-up. Overall survival was calculated using the Kaplan-Meier method and differences between the groups were assessed by the log-rank test. The data are presented as the mean \pm standard deviation. All statistical calculations were performed using SPSS ${ }^{\circledR}$ version 22 (IBM SPSS, Chicago, IL, USA). P $<0.05$ was considered to indicate a statistically significant difference.

\section{Results}

MIB-1 labeling index. The MIB-1 labeling index was $13.4 \pm 15.8$ in patients with non-invasive IPMN and $42.4 \pm 30.3$ in patients with invasive IPMN (Fig. 1). A statistically significant difference was observed between the groups $(\mathrm{P}<0.001)$. A histogram of the MIB labeling index was generated according to the pathological grade (Fig. 2). The labeling index of four patients with invasive IPMN was under 5\% (1 patient, $1 \%$ and 3 patients, $5 \%$ ), while that of three patients with non-invasive IPMN (2 with carcinoma in situ component and 1 with high grade dysplasia) was over the mean labeling index of patients with invasive IPMN.

Performance of MIB-1 labeling index. The receiver operating characteristic curve is shown in Fig. 3. The calculated area under the curve was 0.822 . At a cut-off level set to an index of $15.5 \%$, sensitivity was 0.84 and specificity was 0.79 . This resulted in an accuracy of $81 \%$ and four patients with invasive IPMN would be misdiagnosed. As shown in Fig. 2, the four patients with invasive IPMN exhibited 1, 5, 5 and 5\% in 
Table I. Comparison of various characteristics between patients with non-invasive and invasive intraductal papillary mucinous neoplasm.

\begin{tabular}{|c|c|c|c|}
\hline Characteristic & $\begin{array}{l}\text { Non-invasive } \\
\quad(n=28)\end{array}$ & $\begin{array}{l}\text { Invasive } \\
(\mathrm{n}=25)\end{array}$ & P-value \\
\hline Age $($ mean \pm SD $)$ & $66.0 \pm 8.7$ & $69.4 \pm 9.9$ & 0.190 \\
\hline Gender & & & 0.184 \\
\hline Male & 14 & 17 & \\
\hline Female & 14 & 8 & \\
\hline Involved duct & & & 0.059 \\
\hline Branch & 10 & 3 & \\
\hline Main or Mixed & 18 & 22 & \\
\hline Size & & & 0.743 \\
\hline$<3.0$ & 19 & 18 & \\
\hline$\geq 3.0$ & 9 & 7 & \\
\hline Symptom & & & 0.694 \\
\hline No & 25 & 21 & \\
\hline Yes & 3 & 4 & \\
\hline Main Duct Dilatation & & & 0.509 \\
\hline No & 7 & 4 & \\
\hline Yes & 21 & 21 & \\
\hline Mural Nodule & & & 0.011 \\
\hline No & 25 & 14 & \\
\hline Yes & 3 & 11 & \\
\hline MIB-1 labeling index & & & $<0.001$ \\
\hline$<15.5 \%$ & 25 & 4 & \\
\hline$\geq 15.5 \%$ & 3 & 21 & \\
\hline
\end{tabular}

Bold print denotes statistical significance. SD, standard deviation.

A

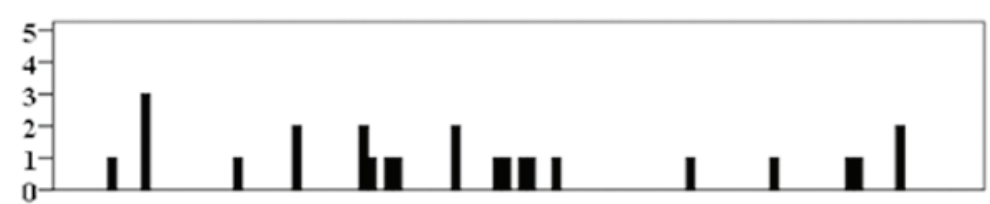

B

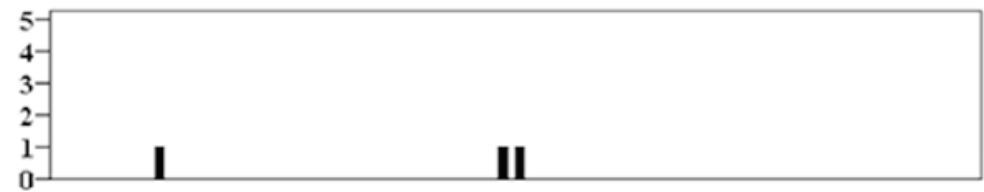

C

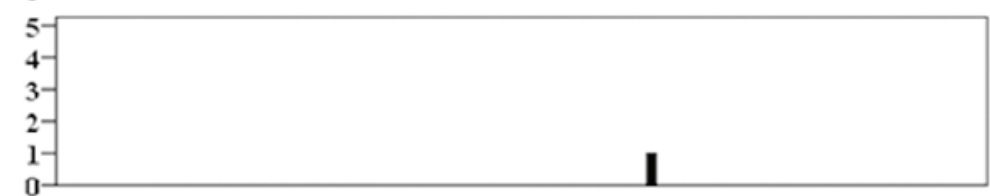

D

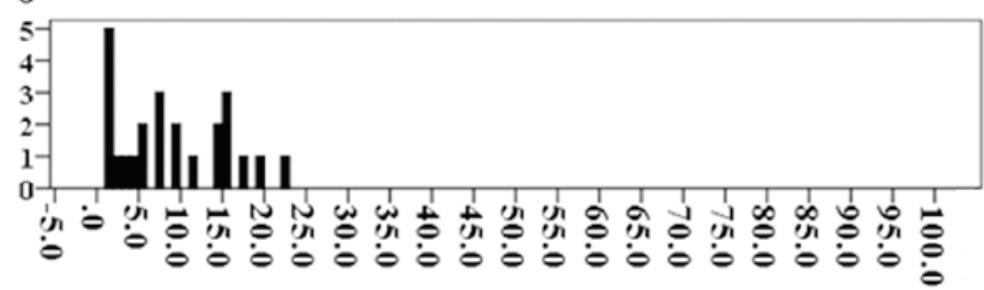

MIB-1 labeling index (\%)

Figure 2. Histogram of the MIB-1 labeling index. (A) Invasive IPMN, and the non-invasive groups, (B) IPMN with carcinoma in situ component, (C) IPMN with high grade dysplasia and (D) IPMN with low grade dysplasia were plotted. IPMN, intraductal papillary mucinous neoplasm. 
Table II. Univariate and multivariate analysis of potential predictive factors for invasive invasive intraductal papillary mucinous neoplasm.

\begin{tabular}{|c|c|c|c|c|c|c|}
\hline \multirow[b]{2}{*}{ Characteristic } & \multicolumn{3}{|c|}{ Univariate analysis } & \multicolumn{3}{|c|}{ Multivariate analysis } \\
\hline & HR & $95 \% \mathrm{CI}$ & P-value & HR & $95 \% \mathrm{CI}$ & P-value \\
\hline Tumor size $(>3 \mathrm{~cm})$ & 0.821 & $0.252-2.670$ & 0.743 & & & \\
\hline Main duct or mixed type & 4.074 & $0.972-17.071$ & 0.055 & & & \\
\hline Symptoms & 1.587 & $0.319-7.905$ & 0.573 & & & \\
\hline Main duct dilatation & 1.750 & $0.445-6.882$ & 0.423 & & & \\
\hline Mural nodule & 6.548 & $1.560-27.484$ & 0.010 & 6.187 & $1.039-36.861$ & 0.045 \\
\hline MIB-1 labeling index $(\geq 15.5 \%)$ & 19.250 & $4.750-78.011$ & $<0.001$ & 18.692 & $4.171-83.760$ & $<0.001$ \\
\hline
\end{tabular}

Bold print denotes statistical significance. HR, hazard ratio; CI, confidential interval.

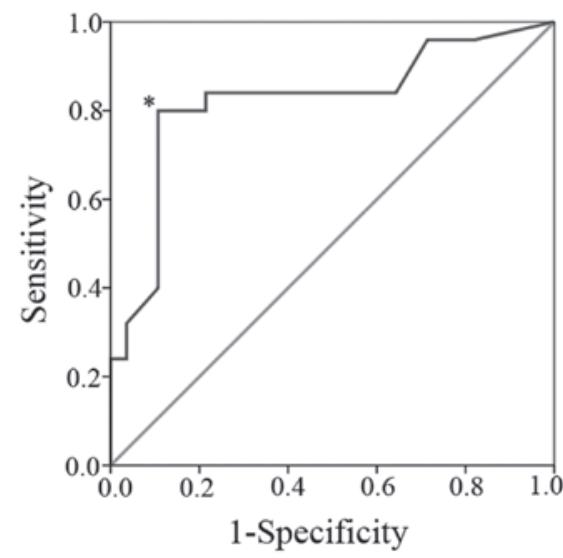

Figure 3. Receiver operating characteristics curve. The calculated area under the curve was $0.822 .{ }^{*}$ The coordinate point when the cut-off threshold of the index was set to $15.5 \%$.

the MIB labeling index. Therefore, it was estimated that the cut-off level of the MIB-1 labeling index be set to $15.5 \%$ as a lower cut-off level was more likely to be inaccurate.

Comparison of characteristics of patients between non-invasive and invasive IPMN. Table I shows the comparison of characteristics of patients between non-invasive and invasive IPMN. The mean ages of patients with non-invasive IPMNs and invasive IPMNs were 66.0 \pm 8.7 and 69.4 \pm 9.9 , respectively. As for the MIB-1 labeling index, patients with $\geq 15.5 \%$ were classified into the higher group and those with $<15.5 \%$ into the lower group. The existence of a mural nodule and a higher MIB-1 labeling index were significantly more frequent in the patients with invasive IPMN compared with those with non-invasive IPMN ( $\mathrm{P}=0.011$ and $\mathrm{P}<0.001$, respectively). No statistically significant difference was observed between non-invasive and invasive IPMN in the other examined characteristics.

Univariate and multivariate analyses of patients with non-invasive or invasive IPMN. To determine which factors are predictors of invasive IPMN, each one was measured using a logistic regression model. The results are shown in Table II. In the univariate analysis, the existence of a mural nodule

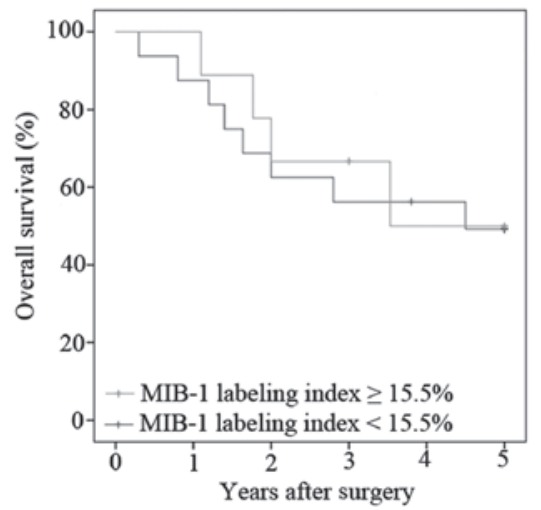

Figure 4. Survival of patients with invasive IPMN according to the level of the MIB-1 labeling index. No statistically significant difference was observed between the groups $(\mathrm{P}=0.798)$. IPMN, intraductal papillary mucinous neoplasm.

and the MIB-1 labeling index achieved statistically significant differences $(\mathrm{P}=0.01$ and $\mathrm{P}<0.001$, respectively). In the multivariate analysis, the existence of a mural nodule (hazard ratio, 6.187; 95\% confidential interval, 1.039-36.861; $\mathrm{P}=0.045$ ) and the MIB-1 labeling (hazard ratio, 18.692; 95\% confidential interval, 4.171-83.760; $\mathrm{P}<0.001)$ were independent predictors of invasive IPMN.

Survival of patients. The MIB-1 labeling index of the patients with invasive IPMN was $43.8 \pm 29.1$. The present study decided to classify the patients into two groups to evaluate prognosis of those with invasive IPMN: Patients with a labeling index $\geq 50 \%$ and the patients with a labeling index $<50 \%$. Fig. 4 shows the overall survival of the patients with invasive IPMN according to the level of the labeling index. The median survival of patients with a lower MIB-1 labeling index was 4.50 years, whereas that of those with a higher index was 3.53 years. No statistically significant differences were observed between the groups $(\mathrm{P}=0.798)$.

\section{Discussion}

The present results revealed that the MIB-1 labeling index and the existence of a mural nodule were predictive factors for 
invasive IPMN. Takeshita et al (22) reported the MIB-1 labeling index as a prognostic factor; however, to the best of our knowledge, this is the first report describing the MIB-1 labeling index as a predictor of invasive IPMNs. The report by Takeshita et al (22) revealed statistically significant differences of the MIB-1 labeling index between low- or intermediate-grade dysplasia and high-grade dysplasia, or carcinoma in situ component, or an invasive component of IPMN (22). Other previous reports have described the MIB-1 labeling index in the context of cell proliferation (15-21). Abe et al (18) reported that the MIB-1 labeling index increased in accordance with adenoma, borderline lesion and carcinoma in situ. However, as a result of the confusing criteria for defining malignant IPMN, it is difficult to compare these results (18). Therefore, the present study classified the patients into two groups: Non-invasive, including IPMNs with carcinoma in situ component, and invasive IPMN. By contrast, the existence of a mural nodule has been reported to be a predictive factor for invasive $\operatorname{IPMN}(6,7)$.

As for the performance of the MIB-1 index as a predictor of invasive IPMNs, the receiver operating characteristic curve proved that an index threshold of $15.5 \%$ was the best to distinguish between non-invasive and invasive IPMN. However, 4/25 patients with invasive IPMN exhibited 1, 5, 5 and 5\% in the MIB labeling index. These patients could therefore not be detected by a lowered cut-off level. Among these patients, three patients exhibited a cyst size $>3.0 \mathrm{~cm}$, main duct dilation, or an abnormal tumor marker. Additionally, two of those exhibtied a mural nodule. They could be detected as high risk for malignancy by the worrisome features and/or high risk stigmata. Therefore, a combination of clinical features, including worrisome features and high risk stigmata with the MIB-1 labeling index would be useful.

In terms of the prognosis of invasive IPMNs, the analysis of the present study revealed no statistical significance between patients with a lower or higher MIB-1 labeling index. Takeshita et al (22) reported that IPMN with low- or intermediate-grade dysplasia exhibited a significantly improved prognosis compared with IPMN with an associated invasive carcinoma, while no statistically significant difference was observed between the prognosis of IPMN with high grade dysplasia and with an associated invasive carcinoma (22). It was also reported that the MIB-1 labeling index significantly increased from $1.8 \%$ in IPMN with low- or intermediate-grade dysplasia to $14-23 \%$ in carcinoma, concluding that a sudden change in proliferative activity occurred between the two. A difference in classification of IPMN existed between the previous report and the present study, which classified high grade dysplasia into non-invasive IPMN.

To use the MIB-1 labeling index as a predictor, pre-operative assessment is required. Fine-needle aspiration biopsy using endoscopic ultrasonography can provide a preoperative opportunity to assess the labeling index. However, due to concerns over intra-abdominal dissemination, fine-needle aspiration is not always performed prior to operation $(23,24)$. The feasibility of assessing the MIB-1 labeling index using fine needle aspiration biopsy under endoscopic ultrasonography must be determined by a clinical research in the future since tumors have heterogeneity in the MIB-1 labeling index.

In conclusion, the MIB-1 labeling index and the existence of mural nodules were proven to be useful as an indicator of invasive IPMN. Although malignancy of certain patients failed to be detected by the MIB-1 labeling index, a combination of worrisome features and high risk stigmata, including the existence of mural nodule) may assist in accurately diagnosing patients with invasive IPMN. The MIB-1 index must be considered as a candidate for the classification of IPMNs.

\section{References}

1. Ohashi K, Murakami Y, Maruyama M, Takekoshi T, Ohta H, Ohhashi I, Takagi K and Kato K: Four cases of mucous secreting pancreatic cancer. Prog Dig Endosc 20: 348-351, 1982.

2. Werner J, Fritz S and Büchler MW: Intraductal papillary mucinous neoplasms of the pancreas-a surgical disease. Nat Rev Gastroenterol Hepatol 9: 253-259, 2012.

3. Tanaka M, Chari S, Adsay V, Fernandez-del Castillo C, Falconi M, Shimizu M, Yamaguchi K, Yamao K and Matsuno S; International Association of Pancreatology: International consensus guidelines for management of intraductal papillary mucinous neoplasms and mucinous cystic neoplasms of the pancreas. Pancreatology 6: 17-32, 2006.

4. Tanaka M, Fernández del Castillo C, Adsay V, Chari S, Falconi M, Jang JY, Kimura W, Levy P, Pitman MB, Schmidt CM, et al: International consensus guidelines 2012 for the management of IPMN and MCN of the pancreas. Pancreatology 12: 183-197, 2012.

5. Salvia R,Fernández-del Castillo C,Bassi C, Thayer SP, Falconi M, Mantovani W, Pederzoli P and Warshaw AL: Main-duct intraductal papillary mucinous neoplasms of the pancreas: Clinical predictors of malignancy and long-term survival following resection. Ann Surg 239: 678-685; discussion 685-687, 2004.

6. Roch AM, Ceppa EP, Al-Haddad MA, DeWitt JM, House MG, Zyromski NJ, Nakeeb A and Schmidt CM: The natural history of main duct-involved, mixed-type intraductal papillary mucinous neoplasms: Parameters predictive of progression. Ann Surg 260: 680-688; discussion 688-690, 2014.

7. Sugiyama M, Izumisato $\mathrm{Y}$, Abe $\mathrm{N}$, Masaki $\mathrm{T}$, Mori $\mathrm{T}$ and Atomi Y: Predictive factors for malignancy in intraductal papillary-mucinous tumors of the pancreas. Br J Surg 90: 1244-1249, 2003.

8. Fritz S, Hackert T, Hinz U, Hartwig W, Büchler MW and Werner J: Role of serum carbohydrate antigen 19-9 and carcinoembryonic antigen in distinguishing between benign and invasive intraductal papillary mucinous neoplasm of the pancreas. Br J Surg 98: 104-110, 2011.

9. Kang MJ, Jang JY, Kim SJ, Lee KB, Ryu JK, Kim YT, Yoon YB and Kim SW: Cyst growth rate predicts malignancy in patients with branch duct intraductal papillary mucinous neoplasms. Clin Gastroenterol Hepatol 9: 87-93, 2011.

10. He J, Cameron JL, Ahuja N, Makary MA, Hirose K, Choti MA, Schulick RD, Hruban RH, Pawlik TM and Wolfgang CL: Is it necessary to follow patients after resection of a benign pancreatic intraductal papillary mucinous neoplasm? J Am Coll Surg 216: 657-665; discussion 665-667, 2013

11. Fritz S, Schirren M, Klauss M, Bergmann F, Hackert T, Hartwig W, Strobel O, Grenacher L, Büchler MW and Werner J: Clinicopathologic characteristics of patients with resected multifocal intraductal papillary mucinous neoplasm of the pancreas. Surgery 152 (3 Suppl 1): S74-S80, 2012.

12. Sturm EC, Roch AM, Shaffer KM, Schmidt CM II, Lee SJ, Zyromski NJ, Pitt HA, Dewitt JM, Al-Haddad MA, Waters JA and Schmidt CM: Obesity increases malignant risk in patients with branch-duct intraductal papillary mucinous neoplasm. Surgery 154: 803-808; discussion 808-809, 2013.

13. Tang LH, Gonen M, Hedvat C, Modlin IM and Klimstra DS: Objective quantification of the Ki67 proliferative index in neuroendocrine tumors of the gastroenteropancreatic system: A comparison of digital image analysis with manual methods. Am J Surg Pathol 36: 1761-1770, 2012.

14. Viale G, Giobbie-Hurder A, Regan MM, Coates AS, Mastropasqua MG, Dell'Orto P, Maiorano E, MacGrogan G, Braye SG, Ohlschlegel C, et al: Prognostic and predictive value of centrally reviewed Ki-67 labeling index in postmenopausal women with endocrine-responsive breast cancer: Results from Breast International Group Trial 1-98 comparing adjuvant tamoxifen with letrozole. J Clin Oncol 26: 5569-5575, 2008. 
15. Semba S, Moriya T, Kimura W and Yamakawa M: Phosphrylated Akt/PKB controls cell growth and apoptosis in intraductal papillary-mucinous tumor and invasive ductal adenocarcinoma of the pancreas. Pancreas 26: 250-257, 2003.

16. Kataoka TR, Ioka T, Tsukamoto Y, Matsumura M, Ishiguro S and Nishizawa Y: Nuclear expression of STAT5 in intraductal papillary mucinous neoplasms of the pancreas. Int J Surg Pathol 15: 277-281,2007.

17. Okada K, Masuda N, Fukai Y, Shimura T, Nishida Y, Hosouchi Y, Kashiwabara K, Nakajima T and Kuwano H: Immunohistochemical expression of 14-3-3 sigma protein in intraductal papillary mucinous tumor and invasive ductal carcinoma of the pancreas. Anticancer Res 26: 3105-3110, 2006.

18. Abe K, Suda K, Arakawa A, Yamasaki S, Sonoue H, Mitani K and Nobukawa B: Different patterns of p16INK4A and p53 protein expressions in intraductal papillary-mucinous neoplasms and pancreatic intraepithelial neoplasia. Pancreas 34: 85-91, 2007.

19. Okimura A, Hirano H, Nishigami T, Ueyama S, Tachibana S, Fukuda Y, Yamanegi K, Ohyama H, Terada N and Nakasho K: Immunohistochemical analysis of $\mathrm{E}$ cadherin, beta-catenin, CD44s, and CD44v6 expressions, and Ki-67 labeling index in intraducta papillary mucinous neoplasms of the pancreas and associated invasive carcinomas. Med Mol Morphol 42: 222-229, 2009.
20. Islam HK, Fujioka Y, Tomidokoro T, Sugiura H, Takahashi T, Kondo $\mathrm{S}$ and Katoh H: Immunohistochemical analysis of expression of molecular biologic factors in intraductal papillary-mucinous tumors of pancreas-diagnostic and biologic significance. Hepatogastroenterology 46: 2599-2605, 1999.

21. Terada T, Ohta T, Kitamura Y, Ashida K and Matsunaga Y: Cell proliferative activity in intraductal papillary-mucinous neoplasms and invasive ductal adenocarcinomas of the pancreas: An immunohistochemical study. Arch Pathol Lab Med 122: 42-46, 1998.

22. Takeshita A, Kimura W, Hirai I, Takasu N, Moriya T, Tezuka K and Watanabe T: Clinicopathologic study of the MIB-1 labeling index (Ki67) and postoperative prognosis for intraductal papillary mucinous neoplasms and ordinary ductal adenocarcinoma. Pancreas 41: 114-120, 2012.

23. Yamao K, Yanagisawa A, Takahashi K, Kimura W, Doi R, Fukushima N, Ohike N, Shimizu M, Hatori T, Nobukawa B, et al: Clinicopathological features and prognosis of mucinous cystic neoplasm with ovarian-type stroma: A multi-institutional study of the Japan pancreas society. Pancreas 40: 67-71, 2011.

24. Hirooka Y, Goto H, Itoh A, Hashimoto S, Niwa K, Ishikawa H, Okada N, Itoh T and Kawashima H: Case of intraductal papillary mucinous tumor in which endosonography-guided fine-needle aspiration biopsy caused dissemination. J Gastroenterol Hepatol 18: 1323-1324, 2003. 